\title{
RECOGNISING HUMAN AND ANIMAL MOVEMENT BY SYMMETRY
}

\author{
James B. Hayfron-Acquah, Mark S. Nixon and John N. Carter \\ University of Southampton, Southampton S017 1BJ, United Kingdom. \\ \{jbha99r|msn|jnc\}@ecs.soton.ac.uk
}

\begin{abstract}
We show how the symmetry of motion can be extracted by using the Generalised Symmetry Operator for analysing motion and for gait recognition. This operator, rather than relying on the borders of a shape or on general appearance, locates features by their symmetrical properties. This approach is reinforced by the view from psychology that human gait is a symmetrical pattern of motion, and by other works. We applied our new method to compare animal gait, and for recognition by gait. Results show that the symmetry properties of gait appear to be unique and can indeed be used for analysis and for recognition. We have so far achieved promising recognition rates of over $95 \%$. Performance analysis also suggests that symmetry enjoys practical advantages such as relative immunity to noise with capability to handle occlusion and as such might prove suitable for applications like clip-database browsing.
\end{abstract}

\section{INTRODUCTION}

Analysing mammalian movement would intuitively appear more demanding than rigid body movement. There are recent studies in computer vision and in psychology which suggest that human gait has symmetrical properties. We capitalise on these to show that we can distinguish human and animal movement and, further, use this to recognise people by the way they walk.

Recognising people automatically, e.g. by face or palm, is of increasing interest. Recently, gait recognition has been added to this domain. As a biometric, gait concerns recognising people by the way they walk. One major advantage of gait over other biometrics (e.g. fingerprints) is that it does not require contact. Advantages of gait in application scenarios are that it is difficult to conceal to disguise whilst maintaining apparently normal movement. Though it could be argued that physical condition factors such as drunkenness, pregnancy and illness can affect motion, these factors are similar in principle to factors affecting other biometrics. The aim of gait recognition is to recognise people regardless of clothing or differing background. There have been studies of gait in other areas, including medical studies, psychological studies, modelling human motion and tracking people. Amongst these, psychologists suggest gait is a symmetrical pattern of motion[3] and that humans perceive gait as unique.

There are already a number of approaches to automatic gait recognition. In the spatio-temporal approach, which is probably the earliest, the gait signature was derived from the spatio-temporal patterns of a walking person[8]. The different patterns of the motions of the head and the legs in translation and time were extracted. The patterns were then processed to determine the motion of the bounding contours from which a five-stick model was fitted. The gait signature was then derived by normalising the fitted model in terms of velocity, that is by linear interpolation, and encouraging $(85 \%)$ recognition rates were achieved.

In [5], optical flow was used to derive the gait signature by analysing the motion content (shape of motion) of a human walking. Generic object-motion characterisation is also another approach where the gait signature is derived from a parametric eigenspace[6] and the approach was applied to a database of seven subjects with ten image sequences each. The recognition rates were $88 \%$ and $100 \%$ for 8 and 16 eigenvectors, respectively. The approach was extended[4] to use canonical analysis, a model free approach to reduce the dimensionality of the input data whilst optimising class separability. Recently, Shutler et al extended statistical gait recognition via temporal moments [11]. This derived statistics with an intimate relationship to gait, with symmetry properties. In other studies, $[7,1]$ pendula modelled the periodic motion of the thigh during walking, again suggesting that analysis of symmetry is suited to gait recognition.

\section{MOTION SYMMETRY AND ITS EXTRACTION}

Symmetry is a fundamental principle and most objects can give rise to a perception of symmetry[10]. An object is said be to symmetric when the response to symmetry operations is invariant. Such operations can only do so when the shape of the object is known in advance, rendering these symmetry operations inefficient. The discrete symmetry operator can estimate symmetricity without the knowledge of the object's shape, unlike feature extraction operators that find a shape by relying on its border. The symmetry transform assigns a symmetry measure to each point in the image and is determined with respect to a given point-symmetry group. It appears that 
the performance of the symmetry transform is not affected by existence of several objects in the scene[10].

To extract the symmetry of moving subjects, the feature templates are extracted from each gait sequence to give a template sequence. The symmetry operator uses the edge map to assign symmetry magnitude and orientation to each image point, accumulated at the midpoint of each pair of image points.

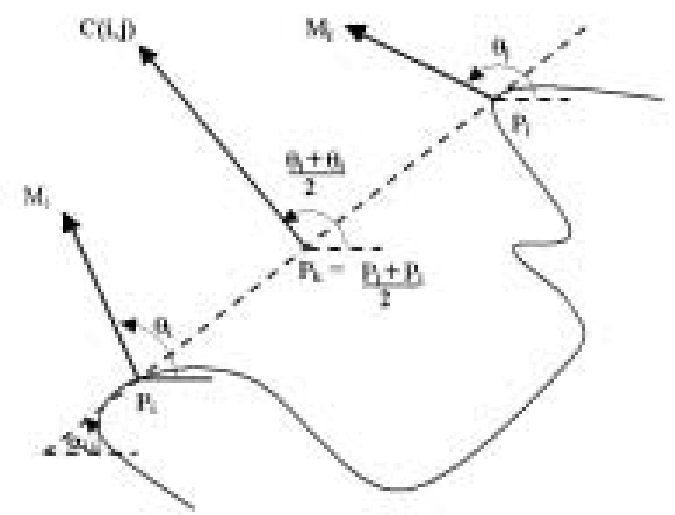

Figure 1 The symmetry contribution of points $P_{i}$ and $P_{j}$

The symmetry distance weighting function, $D$, is defined as the minimum effort required to turn a given shape into its symmetric shape. It reflects the distance between two different points $P_{i}$ and $P_{j}$, and is calculated as:

$$
D_{i, j}=1 / \sqrt{2 \pi \sigma} \exp \left(-\left(\frac{\left\|P_{i}-P_{j}\right\|-\mu}{2 \sigma}\right)\right), \forall i \neq(1)
$$

where $\sigma$ controls the scope of the function. Each value of $\sigma$ implies a different scale thus making it suited to multiresolution schemes. A large value of $\sigma$ implies large-scale symmetry that gives distant points similar weighting to close points. Alternatively, a small value of $\sigma$ implies local operation and local symmetry. Recently a focus, $\mu$, was therefore introduced into the distance weighting function to control the focusing capability of the function, hence further improving the scaling possibilities of the symmetry distance function. The addition of the focus into the distance weighting function moves the attention of the symmetry operator from points close together to a selected distance. The logarithm intensity function, $I_{i}$, of the edge magnitude $M$ (as delivered by, say, the Sobel operator) at point $(x, y)$ is $I_{i}=\log \left(1+M_{i}\right)$. Using the logarithm reduces the differences between high gradients or symmetries resulting from weak edges, making the correlation measure less sensitive to very strong edges. The phase weighting function $P h$ is:

$$
\begin{aligned}
& P h_{i, j}=\left(1-\cos \left(\theta_{i}+\theta_{j}-2 \alpha_{i, j}\right)\left(1-\cos \left(\theta_{i}-\theta_{j}\right)\right),\right. \\
& \forall i \neq j, \text { and } \alpha(i, j)=\operatorname{atan}\left(y_{i}-y_{j} / x_{i}-x_{j}\right)
\end{aligned}
$$

is the angle between the line joining the two points and the horizon. The symmetry relation or contribution, $C(i, j)$ between the two points $P_{i}$ and $P_{j}$ is defined as:

$$
C(i, j)=D_{i, j} P h_{i, j} I_{i} I_{j}
$$

The symmetry contribution value obtained is then plotted at the midpoint $\Gamma(p)$ of the two points, where

$\Gamma\left(p_{k}\right)=\left\{(i, j) \mid \frac{P_{i}+P_{j}}{2}=p_{k}\right\}$

The accumulated symmetry evidence $M \sigma(p)$ for all pairs of points $i$ and $j$ having their midpoint at $p$, Fig. 1, is

$$
M_{\sigma, u}\left(p_{k}\right)=\sum_{(i, j) \in \Gamma\left(p_{k}\right)} C(i, j)
$$

and the direction of contribution of $P_{i}$ and $P_{j}$ is

$$
\varphi(i, j)=\left(\theta_{i}+\theta_{j}\right) / 2
$$

The symmetry transform as discussed here detects reflectional symmetry. It is invariant under $2 \mathrm{D}$ rotation and translation transformations and under change in scale [10], as of potential advantage in gait recognition. In Fig. 2 we show the symmetry map derived for a static elephant. Here, by selection of parameters we can highlight overall symmetry around the centre of mass, Fig. 2c, or local symmetry about the legs (as clearly seen in-between the legs - even between the tusks), Fig. 2d. A combination of these is used in studying the symmetry of motion.

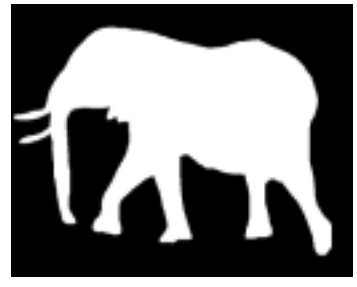

(a) Original

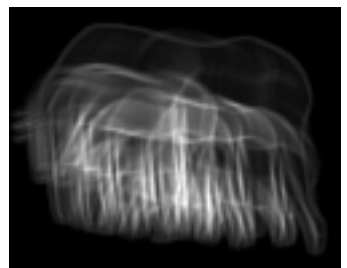

(c) Far-symmetry map

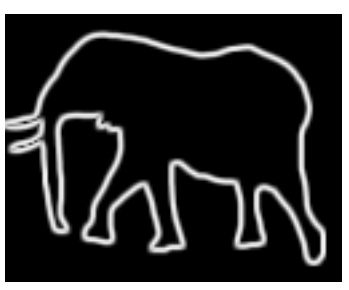

(b) Edge map

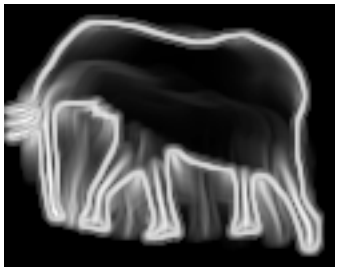

(d) Close-symmetry map
Figure 2 Controlling the action of the symmetry operator

\section{SYMMETRY AND GAIT}

The gait signature for a subject is derived from an image sequence. The following gives an overview of the steps involved. First, the (static) image background is basically subtracted from the original image, Fig. 3a to obtain the silhouette though there are actually new methods for this. The Sobel operator is then applied to the silhouette, Fig. 3b. Where the gait signature is derived from optical flow information, the optical flow image is extracted from two successive silhouettes. The edge-map is thresholded so as to set all points beneath a chosen 
threshold to black, to reduce noise or remove edges with weak strength, as perhaps due to background removal. The symmetry operator is then applied to give the symmetry map, Fig. 3c. For each image sequence, the gait signature is obtained by averaging all the symmetry maps.

The Fourier transform was then applied to each of the gait signatures and the transform was low-pass filtered to ensure the recognition rates were not dominated or influenced by noise.

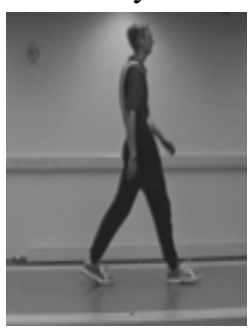

(a) original

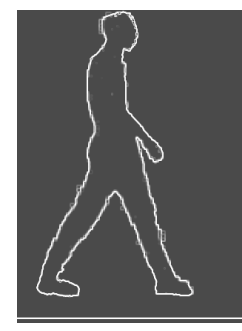

(b) after Sobel

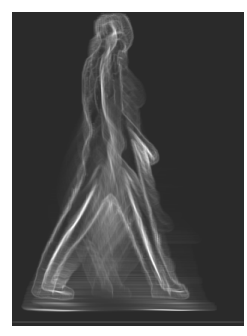

(c) symmetry
Figure 3 Symmetry of Human Silhouette

Different radii were used to determine the effect of selecting part of the Fourier descriptions, as described later. For purposes of classification or recognition, the similarity differences between the Fourier descriptions of the gait signatures are then calculated using Euclidean distance. Considering the Fourier magnitude only gave the best result compared with that by phase information, and was therefore used throughout.

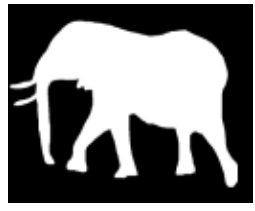

(a) elephant

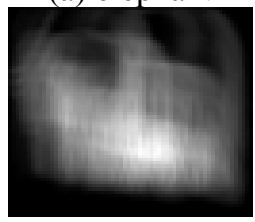

(c) signature for (a)

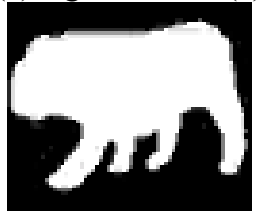

(e) bulldog

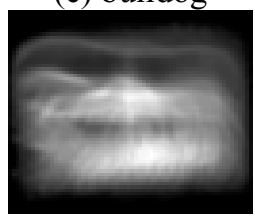

(g) signature for (e)

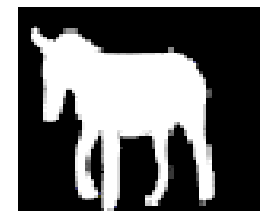

(b) zebra 1

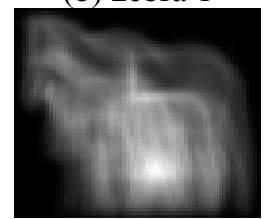

(d) signature for (b)

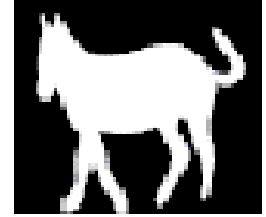

(f) zebra 2

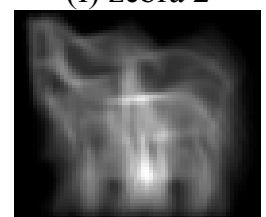

(h) signature for (f)
Figure 4 Symmetry Signatures for Different Animals

\section{RESULTS}

\subsection{Separating animal movement}

The technique was first used to demonstrate that animal movement could be separated. As such the test sequences used were an elephant, a zebra and a bulldog. The concern here is distinction between quadrupeds; the symmetry operator has been arranged specifically to analyse the motion of pairs of legs. As such, this indicates whether symmetry can be used to separate animals by gait.

The gait signatures of these animals are shown in Fig. 4. Here, it can be seen visually that each of these animals is distinct, and by comparison with Fig. 3, different from that of a human. The signatures have two for the same zebra, taken at different times. In one, the tail is moving rapidly but this does not affect the resulting symmetry by the speed of movement and the averaging used. In fact, classification separated each of these sequences perfectly: in terms of structure, the zebra signatures are much closer to each other than the other animals. Naturally, these results are only introductory: future work will aim to qualify how human motion can be separated (not just from similar animal movement), and also how it might be deployed in, say, database browsing.

\subsection{Human recognition by symmetry}

The new method was applied to two different databases of spatial templates. The SOTON database has four subjects with four image sequences each and that of UCSD six subjects with seven image sequences of each. We derived gait signatures for silhouette and optic flow information for both databases as alternative inputs to our method. The values for $\sigma$ and $\mu$ used were 27 and 90, respectively, unless otherwise stated. The $k$-Nearest Neighbour rule was then applied for classification, using $k=1$ and $k=3$, as in Table 1 . The correct classification rates were $100 \%$ for $k=1$ and $k=3$ for the SOTON database. For the UCSD database, the recognition rates for silhouettes were 97.6 and $92.9 \%$ for both values of $k$. A CCR of $92.9 \%$ was obtained for the optical flow information, again for both values of $k$. These encouraging results are similar to those achieved by other techniques on the same data.

\begin{tabular}{|c|c|c|c|c|c|}
\hline \multirow{2}{*}{$\begin{array}{l}\text { Data- } \\
\text { base }\end{array}$} & \multirow{2}{*}{$\begin{array}{c}\text { \# Sub- } \\
\text { jects }\end{array}$} & \multirow{2}{*}{$\begin{array}{c}\text { \# Sequ- } \\
\text { ences }\end{array}$} & \multirow{2}{*}{$\begin{array}{l}\text { Data } \\
\text { Type }\end{array}$} & \multicolumn{2}{|c|}{ CCR (\%) } \\
\hline & & & & $K=1$ & $k=3$ \\
\hline \multirow[b]{2}{*}{ SOTON } & \multirow[b]{2}{*}{4} & \multirow[b]{2}{*}{16} & Silhouette & 100 & 100 \\
\hline & & & Optical flow & 100 & 100 \\
\hline \multirow{2}{*}{ UCSD } & \multirow[b]{2}{*}{6} & \multirow[b]{2}{*}{42} & Silhouette & 97.6 & 92.9 \\
\hline & & & Optical flow & 92.9 & 92.9 \\
\hline
\end{tabular}

Table 1: Initial results obtained from two disparate databases

Even though recognition rates of $100 \%$ were achieved, 
it was observed that selecting fewer Fourier components may affect the recognition rates on a larger database, depending on the value of radius used. This is shown in Fig. 5 where radii less than 4 led to poorer recognition rates. This needs to be investigated further.

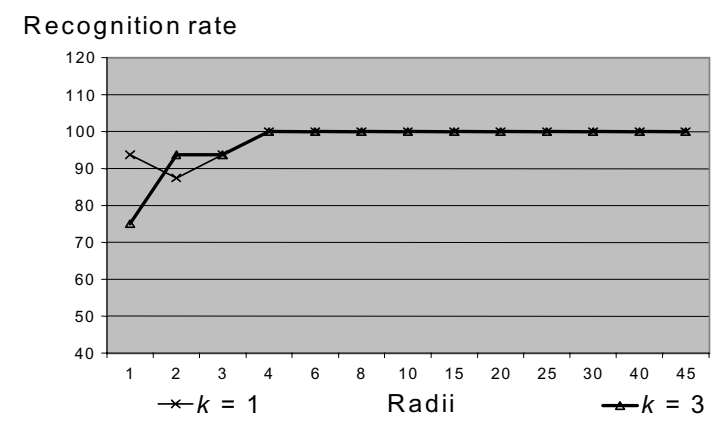

Figure 5 Effect of low pass filtering

\subsection{Performance analysis of symmetry operator}

Performance was evaluated with respect to missing frames, addition/omission of spatial data and noise using the SOTON database. Out of the 16 images sequences in the database, one image sequence of subject 4 was used as the test subject with the remainder for training.

The addition/omission evaluation was done by masking with a rectangular bar of different widths: 5, 10 and 15 pixels in each image frame of the test subject and at the same position. The area masked was on average $13.2 \%$, $26.3 \%$ and $39.5 \%$ of the image silhouettes, respectively. The bar either had the same colour as the image silhouette or as the background, as shown in Fig. 6, simulating omission and addition of data, respectively. In both cases, recognition rates of $100 \%$ were obtained for a bar 5 pixels wide, for both values of $k$. For a bar 10 pixels wide, Fig. $6 \mathrm{c}$ failed but Fig. 6a gave the correct recognition for $k=3$ but not for $k=1$. For bar sizes of 15 and above, the test subject could not be recognised.

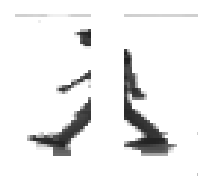

(a) 10 pixels

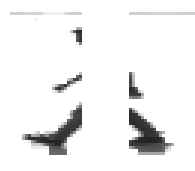

(b) 15 pixels

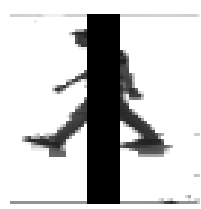

(c) 10 pixels

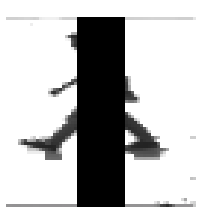

(d) 15 pixels
Figure 6 Occluded Data

Naturally, the symmetry operator can handle noise by the averaging associated with its evidence gathering and experimentation has confirmed that using symmetry can tolerate additive Gaussian noise. The missing image frames evaluation also showed that the operator can handle time lapse imagery. In future we aim to analyse performance in more detail, and on a larger database of subjects.

\section{CONCLUSIONS}

This paper supports the psychology view that the symmetry of motion can be used for recognition. We have therefore presented, as a starting point, a new approach that develops a symmetry signature that can be used to analyse motion and for recognition. The symmetry operator, essentially, forms an accumulator of points, which are measures of the symmetry between image points to give a symmetry map. By using the symmetry operator, the Fourier Transform and a simple classification approach, the results have shown how animal movement can be discriminated, and how human motion can be recognised. Comparable recognition rates for human gait have been achieved using the same databases as in other works. The symmetry operator has performance attributes in that it can handle missing spatial data, missing image frames, and to some extent noise. As such, analysis of motion by symmetry might have a promising future.

\section{REFERENCES}

[1] R. Cutler and L. S. Davis, "Robust Real-Time Periodic Motion Detection: Analysis and Applications", IEEE Trams. PAMI, 21(8), 1999, 781-796

[2] D. Cunado, M. S. Nixon and J. N. Carter, "Gait extraction and description by evidence gathering", Proc. 2nd Int. Conf AVBPA99, Washington, 1999, 43-48

[3] J. T. Cutting, D. R. Proffitt and L. T. Kozlowski, "A biomechanical invariant for gait perception", J. Exp. Psych.: Human Perception and Performance, 1978, 357-372

[4] P. S. Huang, C. J. Harris and M. S. Nixon, "Human gait recognition in canonical space using spatio-temporal templates", IEE Proc. VISP, April 1999, 93-100

[5] J. Little and J. Boyd, "Recognizing People by Their Gait: The Shape of Motion", Videre, 1(2), 1-32, 1998

[6] H. Murase and R. Sakai, "Moving object recognition in eigenspace representation: gait analysis and lip reading", Patt. Recog. Lett., 1996, 155-162

[7] M. S. Nixon et. al., "Automatic Gait Recognition", In: Biometrics Personal Identification in Networked Society, 231250. Kluwer Academic Publishers, 1999

[8] S. A. Niyogi and E. H. Adelson, "Analysing and recognising walking figures in xyt", In: Proc. CVPR, 1994, 469-474

[9] C. J. Parsons and M. S. Nixon, "Introducing focus in the generalised symmetry operator", IEEE SigP Lett., 3, 1999, 49-51 [10] D. Reisfeld, H. Wolfson, and Y. Yeshurun, "Context-free attentional operators: The generalised symmetry transform". IJVC, 1995, 119-130

[11] J. D. Shutler, M. S. Nixon and C. J. Harris, "Statistical gait recognition via temporal moments". 4th IEEE Southwest Symp. on Image Analysis and Int., 2000, 291-295

\section{ACKNOWLEDGEMENTS}

James Ben Hayfron-Acquah gratefully acknowledges tenure of a Commonwealth Research Fellowship and we gratefully acknowledge partial support by the European Research Office of the US Army under Contract No. N68171-01-C-9002. 